\title{
Análisis interpretativo del proceso de acreditación en función del diseño de recomendaciones de gestión académica y directiva en la Facultad de Administración de la Universidad Nacional de Colombia sede Manizales ${ }^{1}$
}

Interpretive analysis of the accreditation process based on the design

of academic and management recommendations at the Faculty of Administration of the National University of Colombia, Manizales headquarters

Recibido: 18 de octubre de 2019 | Aprobado:13 de diciembre de 2019

\section{Resumen}

Este artículo expone el análisis de los informes de acreditación de la Facultad de Administración de la Universidad Nacional de Colombia sede Manizales, de acuerdo a sus modelos de evaluación y al proceso de la evaluación institucional desarrollado por los programas curriculares que la conforman. Además, presenta las estrategias que posibilitan la articulación entre la gestión directiva y académica en el marco del mejoramiento continuo. Este proceso de autoevaluación comprendido como un ejercicio permanente de reflexión y de acción se enmarca en los lineamientos dispuestos por parte del Consejo Nacional de Acreditación de Colombia (CNA) en la "Guía para el mejoramiento institucional”, cuyo propósito es obtener una visión de la situación

* Profesor Asociado de la Facultad de Administración de la Universidad Nacional de Colombia sede Manizales. Investigador Asociado Colciencias Colombia. Para contactar al autor: ubustamantel@unal.edu.co

** Magíster en Administración (Fecha grado 05 de diciembre de 2019). Especialista en Gerencia Estratégica de Proyectos. Coordinador del Sistema de Evaluación Académica en la Dirección Académica de la Universidad Nacional de Colombia sede Manizales. Para contactar al autor: ghhernandezh@unal. edu.co

1 Este artículo se constituye en una síntesis del Trabajo Final de Maestría "Estrategias para la articulación entre la cultura de la evaluación y la gestión directiva y académica con los procesos de autoevaluación y acreditación de los programas curriculares de pregrado de la Facultad de Administración de la Universidad Nacional de Colombia sede Manizales" el cual se encuentra referenciado en el Sistema Nacional de Bibliotecas SINAB de la Vicerrectoría de Investigación de la Universidad Nacional de Colombia y se encuentra en el Repositorio Institucional Bdigital; su consulta puede darse a través del siguiente link: http://bdigital.unal.edu.co/72275. 
actual de los programas curriculares respecto a los criterios de calidad. Esto garantizó la obtención rigurosa de datos para lograr un diagnóstico de los procesos de gestión y administración académica, permitiendo extraer conclusiones y proponer recomendaciones que buscan consolidar una cultura de la autoevaluación y el mejoramiento de los procesos académicos y administrativos. Para este análisis se partió de un estudio inicial exploratorio descriptivo de los factores planteados por la guía del CNA: misión institucional, procesos académicos, organización y gestión, que dan cuenta del estado en que se encuentran los programas curriculares, estableciendo una relación con los procesos de gestión directiva y gestión académica, referentes planteados por el Ministerio de Educación Nacional de Colombia. Desde esta relación se ofrecen recomendaciones académicas y de gestión en el marco del proceso de autoevaluación.

Palabras clave: Cultura de evaluación; acreditación; mejoramiento continuo; gestión directiva; gestión académica.

\section{Abstract}

This paper presents the analysis of the accreditation reports of the Faculty of Administration of the National University of Colombia, Manizales, according to its evaluation models and the process of institutional evaluation developed by the curricular programs that make it up. In addition, it presents the strategies that enable the articulation between managerial and academic management within the framework of continuous improvement. The self-assessment process, understood as a permanent exercise in reflection and action, is part of the guidelines laid down by the National Accreditation Council of Colombia (CNA): A guide to institutional improvement, which aims to provide an overview of the current status of curriculum programmes with respect to quality criteria. This ensured the rigorous collection of data for a diagnosis of management processes and academic administration; from there the data were made available and analysed in a comprehensive and operational manner with reference to the objective and strategic contribution to the self-assessment process, enabling conclusions to be drawn and recommendations to be proposed that seek to consolidate a culture of self-assessment and improve administrative academic processes. It was based on an exploratory descriptive study of the factors raised by the CNA's guide: institutional mission, academic processes, and, organization and management, which give an account of the state of the curriculum, establishing a relationship with the management and academic management processes proposed by the Ministry of National Education of Colombia. From this point of view, academic and management recommendations are proposed within the framework of the self-assessment process.

Keywords: Evaluation culture; accreditation; continuous improvement; management; academic management.

\section{Introducción}

Los modelos y sistemas de aseguramiento de la calidad han cobrado importancia en los últimos años al interior de las Instituciones de Educación Superior (IES) del país, a partir de las directrices del Ministerio de Educación Nacional (MEN), la evaluación y el seguimiento por parte del Consejo Nacional de Acreditación (CNA).

Las Instituciones de Educación Superior (IES) han reconocido los beneficios que pueden obtener al cumplir con estándares de calidad, además de consolidar un proceso de reflexión que sea el resultado de un trabajo responsable y crítico de las comunidades académicas, de forma que el gran esfuerzo institucional y del programa fundamente una cultura de evaluación y el mejoramiento continuo. Sin embargo, en la realidad de las prácticas adelantadas en la autoevaluación de los programas curriculares se han logrado identificar dificultades que ponen en duda el éxito y finalidad de este tipo de procesos.

A partir de la revisión de las experiencias en el desarrollo de los procesos de autoevaluación de los programas curriculares de pregrado de la 
Universidad Nacional de Colombia sede Manizales, fue posible evidenciar que se carece de una gestión académico-administrativa para este tipo de procesos. Es preciso aclarar que la institución cuenta con un sistema de aseguramiento de la calidad robusto desde la definición de la normativa que los rige y los lineamientos propios para adelantar su proceso de principio a fin, así como el desarrollo de plataformas web que facilitan la compilación de la información, como un insumo esencial para la evaluación de cada uno de los factores que estructuran los lineamientos y componentes del proceso de autoevaluación según la guía del CNA. No obstante, es en su implementación en los programas curriculares de la Facultad que se empieza a identificar la necesidad de consolidar una cultura de la evaluación, trasversal a todos los niveles que definen el proceso de autoevaluación.

Este análisis, uno de los resultados de la investigación de maestría titulada "Estrategias para la articulación entre la cultura de la evaluación y la gestión directiva y académica con los procesos de autoevaluación y acreditación de los programas curriculares de pregrado de la Facultad de Administración de la Universidad Nacional de Colombia sede Manizales", indica la relación entre el concepto de gestión directiva y académica establecido por el MEN, y, la "Guía para el mejoramiento institucional" denominada autoevaluación, establecido por el CNA.

Se realizó una descripción de la situación de las prácticas aplicadas (propósitos y actividades para el desarrollo de las etapas) en la autoevaluación con fines de acreditación de los programas curriculares, lo que posibilitó un diagnóstico de los procesos de gestión y administración académica, desde allí se dispusieron y analizaron los datos de manera abarcable y operativa teniendo como referente el objetivo de la investigación y el marco conceptual, lo que facilitó el examen y la interpretación de los datos, permitiendo extraer conclusiones y plantear recomendaciones que permitan la consolidación de una cultura de la autoevaluación y el mejoramiento de sus procesos académico administrativos.

\section{Acercamiento referencial}

En Colombia la Ley 30 de 1992 estableció los referentes que permiten que las IES ofrezcan educación con calidad, para lo cual se organizó el servicio público de la Educación Superior. Como parte de sus obligaciones, las IES se comprometen a "prestar a la comunidad un servicio con calidad, el cual hace referencia a los resultados académicos, a los medios y procesos empleados, a la infraestructura institucional, a las dimensiones cualitativas y cuantitativas del mismo y a las condiciones en que se desarrolla cada institución" (Ley 30, 1992, art. 6), y el Estado se vuelve garante en la inspección y vigilancia a través del "desarrollo de un proceso de evaluación que apoye, fomente y dignifique la Educación Superior" (Ley 30, 1992, art. 32); además, hizo precisión sobre la autonomía que tendrían las Instituciones para adelantar el proceso de evaluación de acuerdo a su naturaleza y especificidades de sus áreas de acción.

En este marco, el CNA ha sido la entidad encargada de brindar garantías para que las IES y sus programas alcancen los niveles más altos de calidad en beneficio de la sociedad, a través de la construcción de lineamientos que orientan a los programas y las mismas instituciones en sus procesos de evaluación interna. Para esto el CNA precisó que a partir de estos lineamientos las mismas instituciones "están en libertad de utilizar sus propios instrumentos para la recolección de información, de definir nuevas características y aspectos a evaluar o de darles lecturas diferenciadas, para efectos de conducir más apropiadamente sus procesos de autoevaluación" (CNA, 2013, p.18), lo que ha permitido que la autoevaluación empiece a ser considerada como un aprendizaje institucional en todos sus niveles.

Para la Universidad Nacional de Colombia (UN) el inicio del siglo XXI se caracterizó por el desarrollo de actividades y la creación de normas en torno a los procesos de autoevaluación y acreditación de sus programas curriculares; analizó las mejores alternativas de evaluación externa y las confrontó con la naturaleza, características y condiciones internas de la Universidad.

Como ejercicio desde las experiencias obtenidas en los diferentes procesos de autoevaluación a través de los años y parte de las reflexiones sobre los beneficios y dificultades presentadas, la Universidad diseñó e implementó su propio modelo, teniendo como referencia los lineamientos del CNA, para 
lo cual ha elaborado diferentes guías de autoevaluación y seguimiento de la calidad de los programas de pregrado y posgrado ${ }^{1}$. Para el año 2019, la Universidad Nacional de Colombia presentó el siguiente panorama de acreditación de sus programas de pregrado y posgrado:

Tabla 1. Programas curriculares acreditados UN

\begin{tabular}{|c|c|c|}
\hline \multirow{2}{*}{ SEDE } & \multicolumn{2}{|c|}{ ACREDITADOS } \\
\cline { 2 - 3 } & PREGRADO & POSGRADO \\
\hline Bogotá & 36 & 40 \\
\hline Manizales & 9 & 5 \\
\hline Medellín & 19 & 16 \\
\hline Palmira & 5 & 2 \\
\hline UN & 69 & 63 \\
\hline
\end{tabular}

Fuente: Dirección Nacional de Programas de Pregrado y Posgrado - UN (2019)

Propiamente para la sede Manizales, el escenario de acreditación de sus programas curriculares es el siguiente:

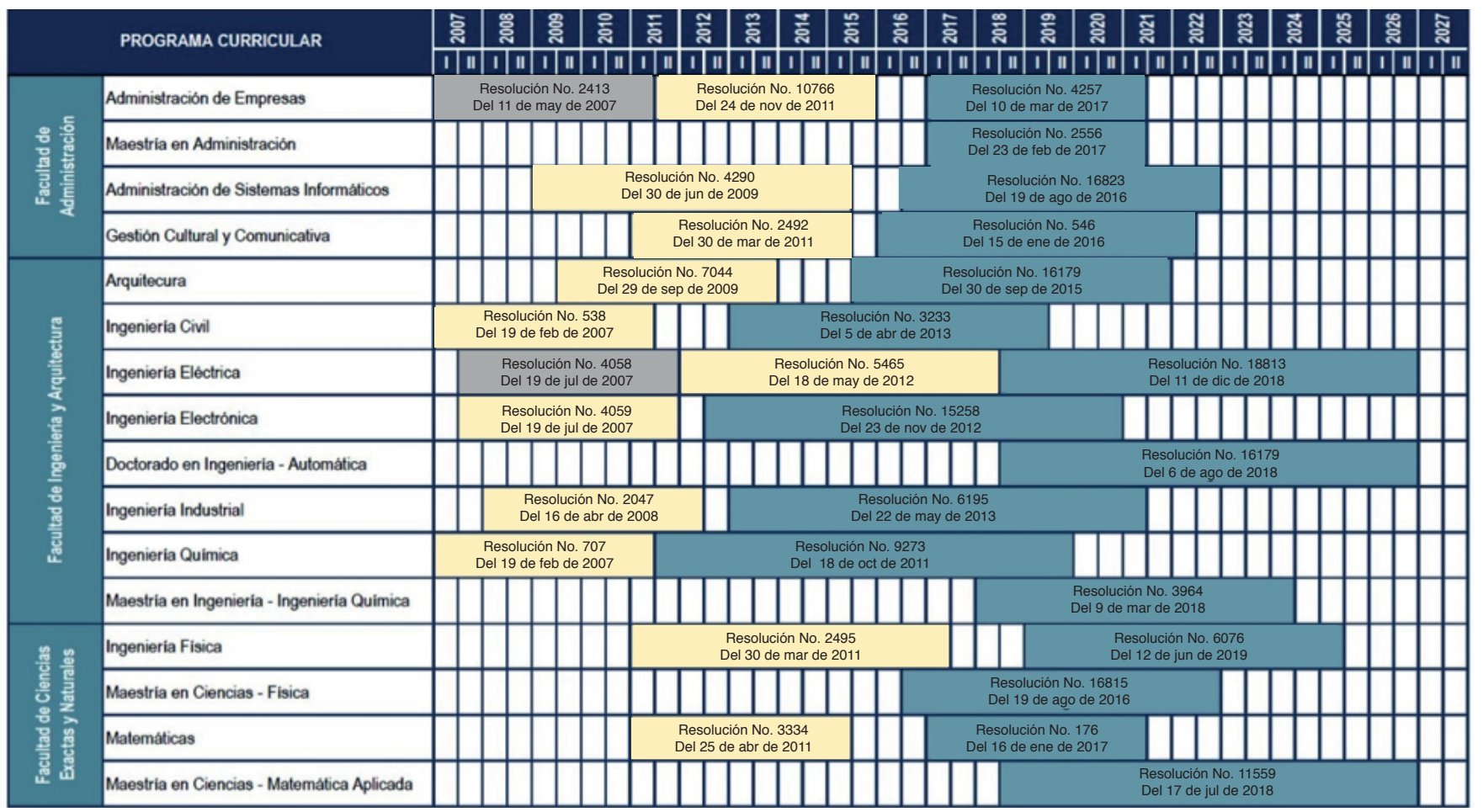

Figura 1. Acreditación de los programas curriculares de la UN sede Manizales. Esta imagen ilustra la resolución de acreditación y el tiempo concedido por el C.N.A. como programa acreditado. El color amarillo corresponde al periodo de la primera acreditación y el color azul a la segunda acreditación. Fuente. Dirección Académica - UN sede Manizales (2019)

En el caso particular de la Facultad de Administración de la sede Manizales, su participación en los procesos de autoevaluación ha permitido que los programas curriculares de pregrado (Administración de Empresas, Administración de Sistemas Informáticos y Gestión Cultural y Comunicativa) cuenten con los más altos estándares de calidad según el C.N.A. 


\section{Una mirada al aseguramiento de la calidad en la educación superior en Colombia}

Las discusiones en torno a los procesos y mecanismos de seguimiento a la evaluación de la educación dadas alrededor del mundo en el siglo XX llevaron a Colombia a considerar dentro de sus políticas públicas el diseño e implementación de estándares mínimos que regulen la calidad de los programas curriculares y las mismas IES.

Es así como el Estado por medio de la Ley 30 de 1992 definió el modelo teórico que dio como resultado la formulación de estándares de calidad para la creación y funcionamiento de programas de pregrado (MEN, 2013, p. 37). Para lograrlo, el Estado creó el SNA cuyo objetivo fue "garantizar a la sociedad que las instituciones que hacen parte del Sistema cumplen los más altos requisitos de calidad" (Ley 30, 1992, art. 53). Para Camisón, Cruz y González (2006), el hecho de que exista una variedad de definiciones sobre calidad, más que un problema, ha permitido incluir nuevas variables que enriquecen el concepto.

En el contexto de las IES, el aporte del concepto de calidad ha llevado a "generar reformas curriculares tendientes a mejorar la oferta educativa en general" (González, 2005, p.11), lo cual permite resaltar en este caso el concepto de calidad, no como un fin, sino como un medio para el mejoramiento continuo de las instituciones académicas. En el Artículo 2.5.3.2.1.1. del Decreto 1330 de 2019 el MEN concibe la calidad como "el conjunto de atributos articulados, interdependientes, dinámicos, construidos por la comunidad académica como referentes y que responden a las demandas sociales, culturales y ambientales".

Para la Universidad Nacional de Colombia (2010), teniendo como referente la concepción de calidad dada institucionalmente por el MEN, el que se hace operativo gracias al S.N.A, la calidad es la "representación concreta de los ideales institucionales referentes a la estructura, los procesos y resultados en el cumplimiento de las funciones misionales" (p.8), la cual es concebida de forma transversal a través de los mismos procesos de autoevaluación.
Para el MEN (2010) el proceso autoevaluativo de las IES es la forma objetiva y pública de garantizar su compromiso con el mejoramiento continuo de la calidad, representado en una oferta académica pertinente para la sociedad. De acuerdo con González (1997), a través de la autorregulación universitaria "las instituciones pueden y deben garantizar el mantenimiento de las mejores condiciones de funcionamiento y operación" (p.25); lo que representa para la sociedad misma el mejoramiento de sus condiciones al ser de igual forma garantes en la verificación del quehacer de las IES y sus programas y proyectos educativos.

modelo de aseguramiento de la calidad académica del CNA, asumido por la Universidad para el proceso de evaluación de las instituciones y los programas curriculares, confluye en la articulación y trazabilidad de factores, características y aspectos o indicadores. En sus "Lineamientos para la acreditación de programas de pregrado" del año 2013, los factores establecidos en el modelo de acreditación son agrupados en torno a cuatro dinámicas: diga lo que hace, haga lo que dice, pruébelo y mejórelo.

Para el CNA, los factores son grandes áreas de evaluación que permiten realizar una apreciación sobre las condiciones en las que se encuentra un programa curricular. De manera particular, el CNA ha definido los siguientes factores, reflexionados e implementados actualmente en todas las IES: (1) Misión, Visión y Proyecto Institucional y de Programa, (2) Estudiantes, (3) Profesores, (4) Procesos académicos, (5) Investigación y creación artística y cultural, (6) Visibilidad nacional e internacional, (7) Impacto de los egresados sobre el medio, (8) Bienestar institucional, (9) Organización, administración y gestión, (10) Recursos físicos y financieros.

Para dar razón de estos factores, la Universidad Nacional asumen una serie de etapas que permiten dar alcance a los objetivos de mejoramiento en los programas y las mismas instituciones. La Dirección Académica de la Universidad Nacional de Colombia sede Manizales ha distinguido una serie de etapas de forma cíclica que permite dar seguimiento a los procesos de autoevaluación con fines de acreditación: 


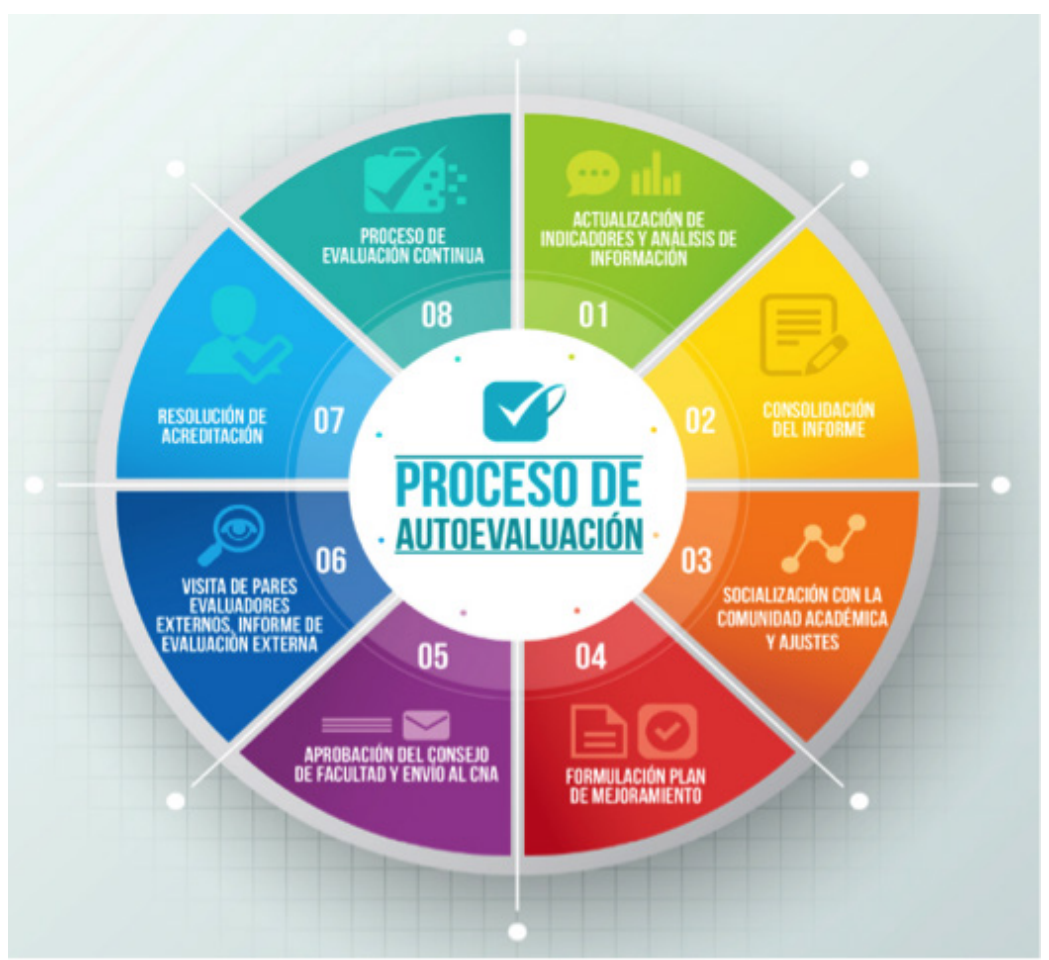

Figura 2. Etapas de un proceso de autoevaluación con fines de acreditación adoptadas por UN. Fuente: Dirección Académica - UN sede Manizales (2019)

Es así como la Universidad Nacional de Colombia desde los referentes establecidos por el MEN respecto a la calidad, genera los espacios de reflexión y evaluación en consonancia con los objetivos instaurados desde el CNA (como entidad asesora) y el MEN, como la instancia que propende por la calidad y excelencia de los procesos de gestión académica de las diferentes instituciones del país, fortaleciedo poco a poco y desde su proceso una cultura de la calidad de la autoevaluación.

El MEN, a través del Decreto 1295 de 2010, fomenta la existencia de una cultura de autoevaluación que contemple el diseño e implementación de políticas que hagan partícipe a la comunidad académica de una institución. En este marco referencial, los lineamientos para la acreditación propuestos por el CNA, de acuerdo con Pabón Fernández (2001), "se enmarcan en un planteamiento de compromiso con la calidad, que reconoce la vocación y la identidad de las instituciones de educación superior (IES), con fundamento en la afirmación de la pluralidad y el respeto por la diversidad" (p. 3), exaltando así estos aspectos como parte primordial en una cultura de la evaluación-autoevaluación propia de cada institución.

Para Bolseguí y Fuguet (2006), "la cultura de cada institución se genera a partir de las estructuras, roles, códigos de conducta, normas, patrones de acción y comunicación" (p. 7). Por su parte, Mabawonku (2003), citado por Ehlers (2010), interpreta la cultura como aquellos propósitos y herramientas que se generan para alcanzar los objetivos del grupo o comunidad. De esta forma, es por medio de la interacción en la comunidad académica que se construye su identidad, su forma de accionar, su particularidad y diferenciación entre las demás; así, la cultura puede ser propicia para determinar la forma de gestión tanto administrativa como académica.

A partir de los propósitos que enmarcan la cultura, esta se origina y se hace única e irrepetible. Sin embargo, es a partir de las personas y sus relaciones al interior de una institución que se hace particular; en el caso de las instituciones educativas, la gestión de los procesos educativos como la enseñanza, el aprendizaje, la evaluación y el funcionamiento de 
la institución son parte de los insumos necesarios para la construcción de su cultura, de su identidad.

La evaluación, desde el referente educativo institucional que asume la Universidad, relata procesos académicos y de gestión; sin embargo, se puede igualmente pensar en el concepto como algo restrictivo; de acuerdo con García (2000), citado por Bolseguí y Fuguet (2006), predomina el hecho de pensar en la idea de evaluación como medición, a través de un corte técnico donde prima el control. No obstante, el concepto de evaluación ha evolucionado a través de la historia y puede ser considerada dependiendo del acercamiento a problemas sociales. En el contexto educativo, hasta hace poco, según Reyes (2006), el objeto de evaluación que ha predominado ha sido el estudiantado, pero se han originado investigaciones hacia otros objetos de la evaluación en educación superior como lo son los docentes, los programas formativos y la evaluación institucional.

Para Lecointe (2001), citado por Rangel (2010), "la evaluación debe entenderse como una serie de acciones que propicien una dinámica movilizadora" (p. 1). Así como se da en la cultura, la participación de la comunidad que hace parte de una institución exalta el valor que tiene la ejecución de actividades que evalúen el funcionamiento de la misma. Es así como "la evaluación se convierte en una nueva cultura de participación, de carácter progresivo, que evoluciona continuamente" (Bolseguí y Fuguet, 2006, p. 6), y es quien conlleva a una construcción social que implica cambios en las instituciones.

En este punto, "una cultura de evaluación podría definirse, entonces, como el conjunto de valores, acuerdos, tradiciones, creencias y pensamientos que una comunidad educativa asigna a la acción de evaluación" (Valenzuela, Ramírez y Alfaro, 2011, p. 45). Su fomento debe considerar iniciativas de autoevaluación constantes para el logro efectivo de los procesos de gestión institucional. De ahí que, "la generación y sostenimiento de la cultura de la autoevaluación en las instituciones es la base o cimiento para el aseguramiento de las condiciones mínimas de calidad" (Londoño y Ramírez, 2012, p. 3). Empero, es a partir de la misma gestión directiva y académica que se puede asegurar o no los resultados esperados al adelantar este tipo de procesos. Es necesario que no se llegue al hecho de que "las instituciones puedan aprender el juego de la evaluación/acreditación y desarrollar una cultura de la conformidad" (Orozco y Cardoso, 2003, p. 78).

Casallas y Gaona (2013) van más allá al considerar que la cultura implica una interdependencia de las personas en sus relaciones a través de los diferentes niveles jerárquicos de la institución y las reglas establecidas por estas que hacen que se genere una influencia en las conductas de los individuos que las componen. Es decir, "culture influences an organization through the people within it" (Masland, 1985, p. 158).

La relación que se puede derivar de la cultura y la gestión en una institución puede proporcionar, como lo dice Masland (1985), un fundamento subyacente para el desarrollo institucional, al establecer referentes y parámetros que articulen su realidad y permita proyectar sus objetivos, políticas y estrategias. La comunidad da origen a la cultura en una organización y depende de la gestión de su cultura y la evaluación de su gestión que puede contemplar mantenerse en el tiempo.

Los esfuerzos al interior de una institución de educación superior no deben ser direccionados al cumplimiento de requerimientos normativos, más bien deben contemplar el mejoramiento de su desempeño. De esta forma, la generación de diversos ambientes de reflexión y discusión entre los miembros de la comunidad debe dar como resultado el diseño e implementación de recomendaciones de gestión que consoliden una cultura del mejoramiento de las condiciones de calidad de un programa curricular de la misma institución y las dependencias que la componen.

\section{Sobre el proceso metodológico}

Establecer enfoques metodológicos y técnicos (herramientas) que posibiliten el diagnóstico de los procesos directivos y académicos es una cuestión inusual, no porque antes no se haya emprendido tal tarea, sino por tratarse ahora en un ambiente generalizado en el que incluso participan saberes y profesionales que, por lo general, habían decidido permanecer aislados de la discusión sobre el sentido y la perspectiva que despliegan los procesos de autoevaluación. Ello exige plantear cuestiones centrales: 1) ¿cuáles son los 
fundamentos que constituyen la metodología que posibilite el diagnóstico de los procesos directivos y académicos?, 2) ¿cuáles son las perspectivas que dibuja la autoevaluación? 3) ¿qué perspectivas implican y demandan propósitos prácticos que posibiliten proponer estrategias de gestión directiva y académica?

El marco de referencia es el diagnóstico que aporta el proceso de autoevaluación emanado de la implementación de la guía propuesta por el CNA, la cual se compone de tres categorías:

1. Factor: área grande de desarrollo con que cuenta el programa académico en su que-hacer; es un elemento constitutivo del programa de formación necesario para dar cumplimiento a sus funciones sustantivas.

2. Característica: es un aspecto que describe un factor y determina su calidad permitiendo la diferenciación de un factor con otro. Reúne a su vez, un conjunto de indicadores que tiene relación con el objeto de la misma.

3. Indicador: sirve como medida o señal para conocer y describir una característica.

EI MEN (2008) conviene que la gestión en una institución educativa debe estar integrada por cuatro áreas: 1) Gestión directiva: hace referencia a la manera como es orientada la institución, centrando su accionar en el direccionamiento estratégico, la cultura institucional, la gobernabilidad y la relación con el entorno; 2) Gestión académica: implica sus acciones en el proceso de enseñanza-aprendizaje que permite una evaluación del desempeño integral de los estudiantes de la institución; 3) Gestión administrativa y financiera: brinda, a través de sus procesos de apoyo, el soporte al trabajo institucional de la gestión; 4) Gestión de la comunidad: hace alusión a las relaciones que la institución debe mantener con el entorno.

Para este análisis interpretativo del proceso de acreditación en función del diseño de estrategias, se consideraron las áreas de la gestión directiva y académica (ver Tabla 2), que al ser evaluadas periódicamente permiten la identificación de debilidades y oportunidades que sirven como insumo, a lo que el MEN denomina "ruta del mejoramiento". Esta ruta conduce al mejoramiento de las instituciones a través de tres etapas: la autoevaluación institucional, la formulación de planes de mejoramiento y el seguimiento a los planes. Estas dos áreas buscan ser articuladas con los factores establecidos en los procesos de autoevaluación del modelo de acreditación del CNA.

Tabla 2. Procesos y componentes de las áreas de gestión institucional

\begin{tabular}{|l|l|l|}
\hline \multirow{2}{*}{ Área } & Proceso & Componentes \\
\hline \multirow{2}{*}{ Directiva } & $\begin{array}{l}\text { Direccionamiento } \\
\text { estratégico y horizonte } \\
\text { institucional }\end{array}$ & $\begin{array}{l}\text { Planteamiento estratégico: misión, visión, valores institucionales (principios), } \\
\text { metas, conocimiento y apropiación del direccionamiento, política de inclusión } \\
\text { de personas con capacidades disímiles y diversidad cultural. }\end{array}$ \\
\cline { 2 - 3 } & $\begin{array}{l}\text { Gestión } \\
\text { estratégica }\end{array}$ & $\begin{array}{l}\text { Liderazgo, articulación de planes, proyectos y acciones, estrategia pedagógica, } \\
\text { uso de información (interna y externa) para la toma de decisiones, seguimiento } \\
\text { y autoevaluación. }\end{array}$ \\
\hline \multirow{2}{*}{ Académica } & $\begin{array}{l}\text { Diseño } \\
\text { pedagógico } \\
\text { (curricular) }\end{array}$ & $\begin{array}{l}\text { Plan de estudios, enfoque metodológico, recursos para el aprendizaje, } \\
\text { evaluación. }\end{array}$ \\
\hline
\end{tabular}

Fuente: Elaboración propia a partir del MEN (2008) 
De los procesos de la gestión directiva establecidos por el MEN se consideraron dos como fundamentales para la revisión y comparación con el ejercicio de autoevaluación y establecidos en la guía del CNA: 1) direccionamiento estratégico y horizonte institucional y, 2) gestión estratégica. El proceso direccionamiento estratégico y horizonte institucional establece los lineamientos que orientan la acción institucional en cada área de trabajo. Por su parte, la gestión estratégica proporciona la oportunidad de tomar decisiones en momentos críticos, pero intenta asegurar, además, que la estrategia sea implementada.

\section{Estrategias de gestión directiva y académica: una mirada desde la autoevaluación.}

Para Montoya (2009), "la estrategia le permite un mejor ejercicio de adaptación a la organización para que se ajuste al entorno y de esa manera la organización evolucione" (p. 32). Desde el ámbito organizacional, la estrategia es entendida, según Mintzberg (1997), como "el patrón de una serie de acciones que ocurren en el tiempo" (p. 3). En cuanto a Gavetti y Levinthal (2000), citados por Montoya (2009), establecen que el "el proceso de formación de la estrategia está vinculado con la acumulación de experiencia" (p. 32). Entonces, a través de la misma madurez institucional y la evaluación de las prácticas desarrolladas por la organización es posible identificar la mejor forma de establecer e implementar sus objetivos de acuerdo a su realidad y a su entorno, involucrando así procesos, actividades, recursos y responsables que a mediano y largo plazo cumplan con las estrategias implementadas.

Ahora bien, la gestión académica, según el MEN, como segunda área de la gestión institucional "es la esencia del trabajo de un establecimiento educativo" (2008, p. 27). Esta área de gestión es la que permite definir las acciones que se enfocan en el desarrollo de competencias y habilidades de los estudiantes en su proceso de aprendizaje. El principal proceso que contiene esta área es la que considera el diseño pedagógico (curricular) como el medio para que el estudiante aprenda en el tiempo y con las herramientas necesarias.

El currículo o plan de estudio, de acuerdo con Ferrer (2008), es el "elemento articulador de los procesos de enseñanza y aprendizaje" (pp. 283-284). Su componente da cuenta de las necesidades metodológicas que dan origen a la relación estudiante - profesor y a la evaluación de las iniciativas de aprendizaje de los estudiantes y de enseñanza de los profesores.

Gimeno Sacristán (1998) considera que el "curriculum" es el contenido y la guía que determina el progreso del alumno en su permanencia en la institución, considerando no solo las determinaciones temáticas de cualquier plan, sino los medios para el desarrollo de la práctica educativa y la forma de evaluación de competencias y habilidades de los estudiantes. Sin embargo, el mayor reto es lograr abrir un espacio a los aspectos académicos entre las líneas estratégicas de las IES, que arraigue sentidos de pertenencia frente a las propuestas de mejoramiento de la calidad de la academia, pero reflejadas en resultados tangibles de los mismos procesos adelantados.

Para encontrar una relación entre la gestión directiva y académica contemplada por el MEN con los procesos de autoevaluación adelantados por los programas curriculares se tomaron algunos de los factores y características establecidos por el CNA. El primer acercamiento se dio entre el factor 1 - "Misión, Visión y Proyecto Institucional y de Programa" del proceso de autoevaluación propuesto por el CNA y el proceso de "direccionamiento estratégico y horizonte institucional" del área de Gestión Directiva establecido por el MEN como parte de la guía de mejoramiento institucional.

El factor "Misión, Visión y Proyecto Institucional y de Programa" permite llevar a cabo una revisión de los propósitos o fines fundamentales para una buena gestión institucional. Para el CNA (2013) este factor permite a un programa curricular ser reconocido por tener un proyecto educativo (PEP) que sea coherente y consecuente con el proyecto educativo institucional. El PEP es la brújula que contiene los "lineamientos, las políticas y los principios que 
Tabla 3. Relación entre gestión directiva (MEN) y factores del proceso de autoevaluación (CNA) - (1)

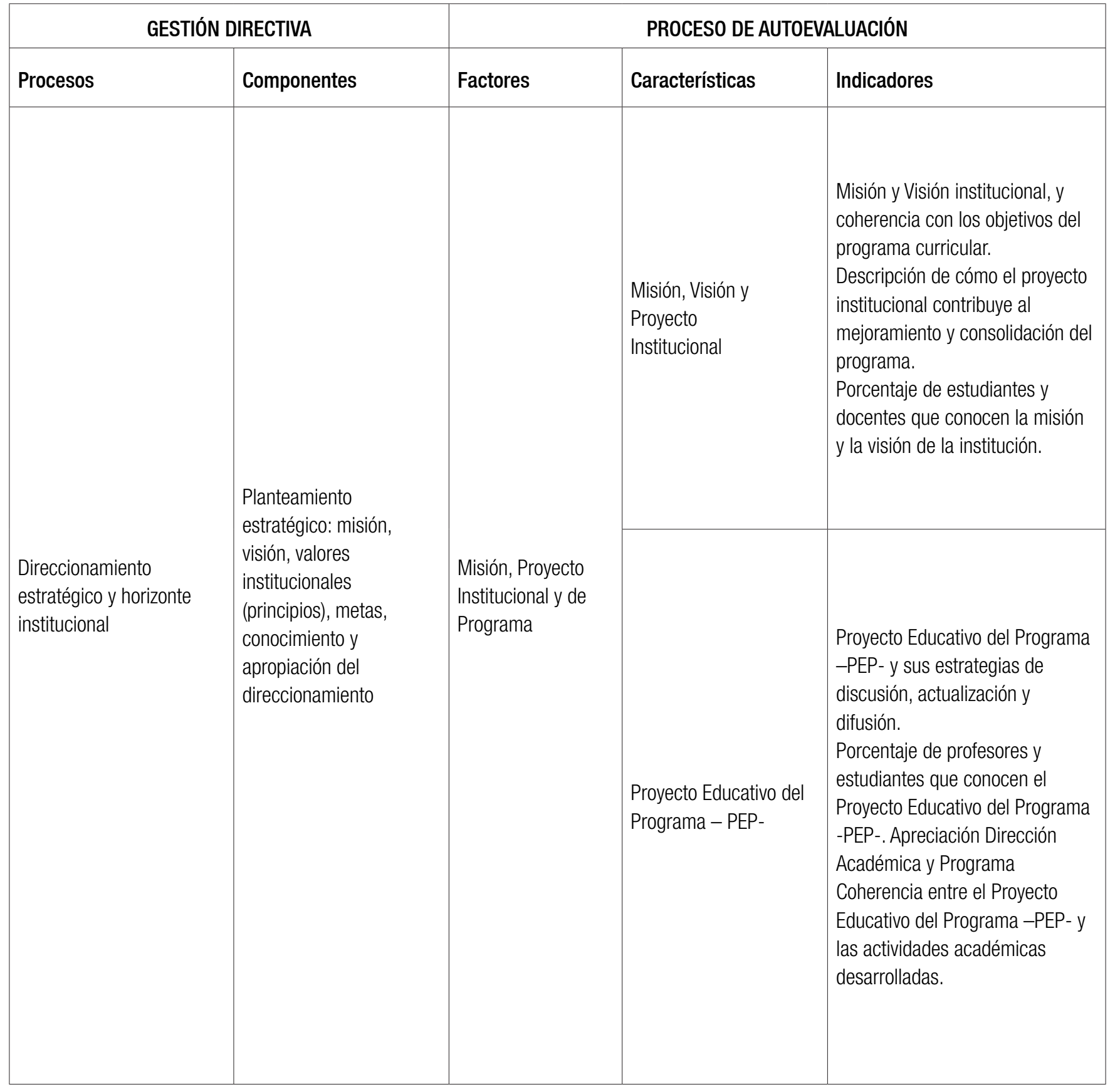

orientan y dirigen el desarrollo del programa" (Universidad Nacional de Colombia, 2012, p. 2). De esta forma, se consideraron las características propias de este factor: "misión, visión y proyecto institucional" y "proyecto educativo del programa - PEP".

Por su parte, en el área de la Gestión Directiva, el proceso de "direccionamiento estratégico y horizonte institucional" establece los lineamientos que orientan la acción institucional en todos y cada uno de sus ámbitos de trabajo. De este proceso hace parte el componente de planteamiento estratégico visto como la misión, valores institucionales, metas, conocimiento y apropiación del direccionamiento.

El segundo acercamiento se da entre el factor 4 "procesos académicos" y el factor 9 "Organización, administración y gestión" correspondiente del proceso de autoevaluación del CNA, con el proceso de "gestión estratégica" del área de Gestión Directiva. 
Tabla 4. Relación entre gestión directiva (MEN) y factores del proceso de autoevaluación (CNA) - (2)

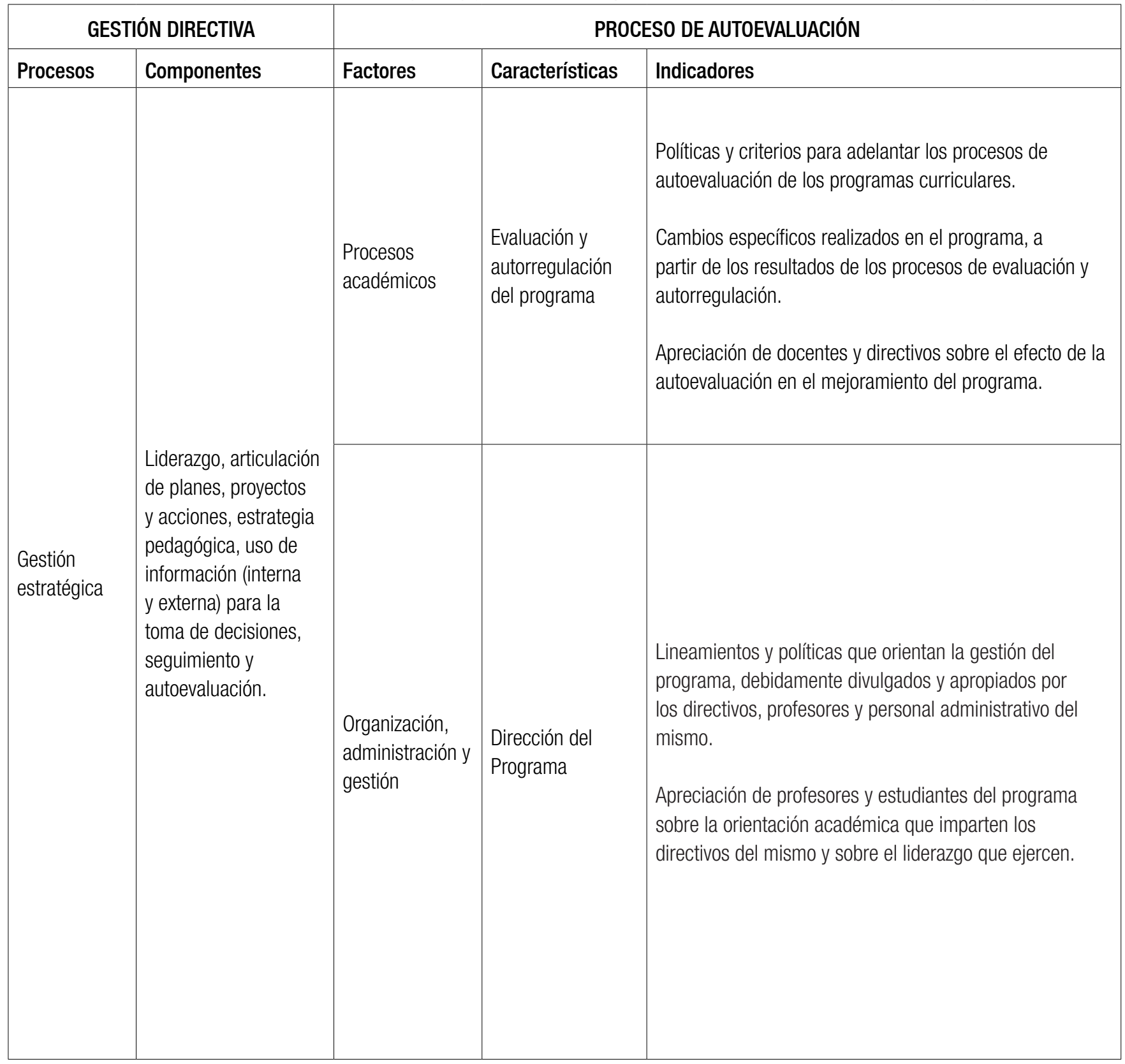

El MEN considera que el proceso de gestión estratégica a través del liderazgo, el uso de información para la toma de decisiones y el seguimiento y autoevaluación pueden brindar las herramientas esenciales para liderar, articular y coordinar todas las acciones institucionales.

Por su parte, el CNA contempla la característica "evaluación y autorregulación del programa" como el espacio en el que se puedan generar las pautas para establecer y liderar estrategias que conduzcan a la implementación de su proyecto educativo. Además, a través de la característica "dirección del programa", el CNA busca establecer la existencia de orientación y liderazgo en la gestión del programa, cuyos métodos de gestión estén claramente definidos y sean conocidos por la comunidad académica.

Una última relación encontrada en el estudio se presenta entre el proceso de "Diseño pedagógico" del área de Gestión Académica del MEN, con el factor 4 "procesos Académicos" del CNA. 
Tabla 5. Relación entre gestión académica (MEN) y factores del proceso de autoevaluación (CNA)

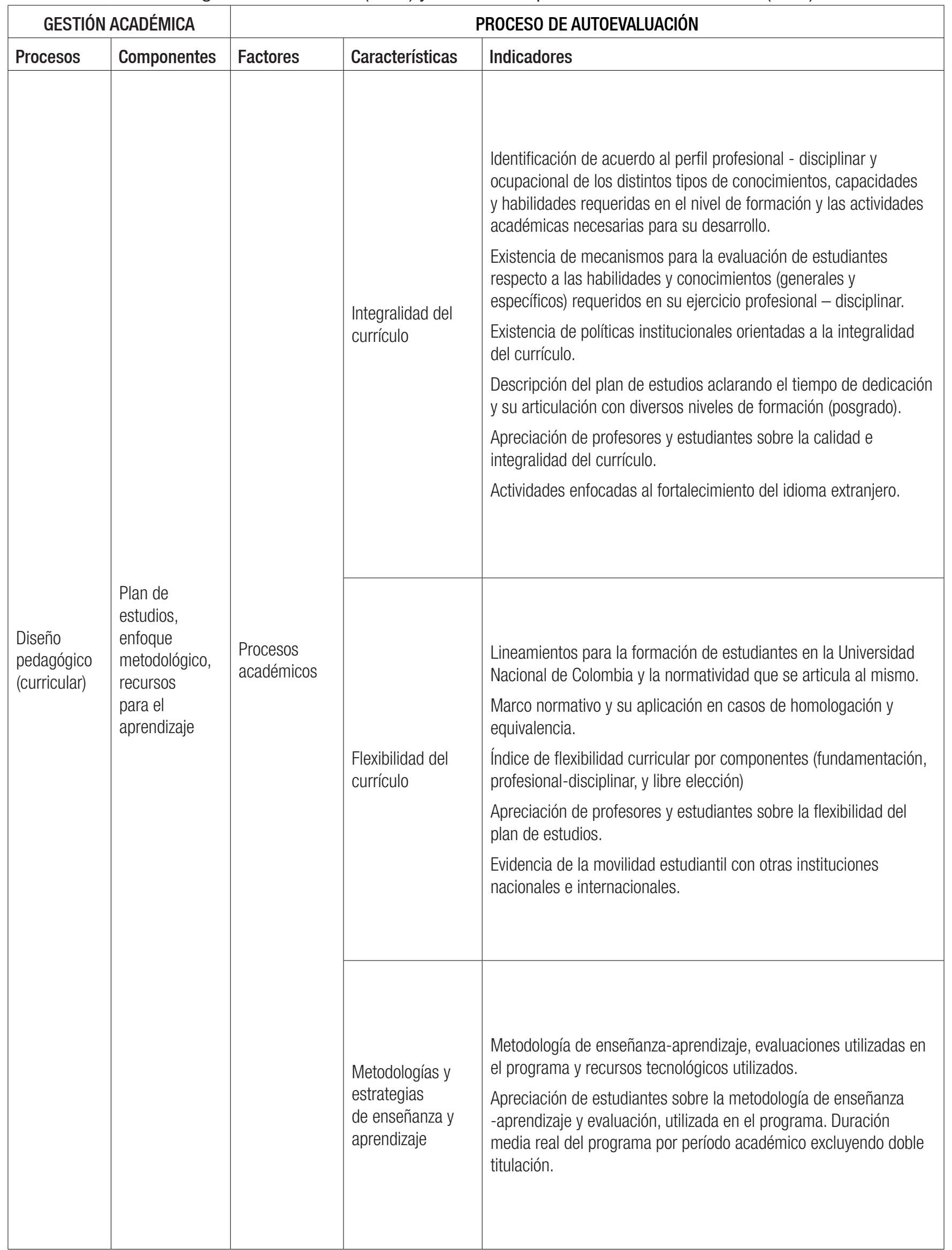


La Gestión Académica considera el "diseño pedagógico" fundamental para determinar lo que los estudiantes deben aprender en cada área o asignatura, el momento, los recursos a emplear y la forma de evaluar sus aprendizajes.

En esta misma línea, el proceso de autoevaluación aporta a la gestión académica desde el factor "procesos académicos", el cual está compuesto por las características "integralidad del currículo", "flexibilidad del currículo" y "metodologías y estrategias de enseñanza y aprendizaje”. La primera intenta establecer cómo el currículo contribuye a la formación de un estudiante integral en sintonía con el Proyecto Educativo del Programa. La segunda característica busca resaltar su dinamismo para conservarse actualizado y pertinente; y la tercera característica identifica los métodos pedagógicos que emplea el programa curricular para el desarrollo de su plan de estudios.

Producto de esos ejercicios de discusión, análisis y evaluación interna, la Universidad estableció en el año 2010 dos documentos de referencia: la "Guía de autoevaluación y seguimiento de la calidad de los programas de pregrado" de la DNPPre, y la guía denominada "Procedimientos para la evaluación de los programas de posgrado con miras al mejoramiento continuo" de la DNPPos.

En el año 2013 la DNPPre publicó la segunda edición de su guía y en el año 2016 la actualizaría la DNPPos tomando como referencia el Acuerdo 151 de 2014 del Consejo Superior Universitario. A través de este Acuerdo, la Universidad normalizó el proceso de autoevaluación y seguimiento de la calidad de sus programas curriculares y definió como etapas fundamentales para cumplir con ese cometido: (i) la Autoevaluación, (ii) la Formulación de Planes de Mejoramiento, (iii) el Seguimiento a Planes de Mejoramiento y (iv) la Evaluación Continua.

En Universidad Nacional de Colombia sede Manizales, desde el acompañamiento brindado por la Vicedecanatura de la Facultad de Administración, desde las prácticas adelantadas por sus programas de pregrado en los procesos de autoevaluación, y conforme al acompañamiento y asesoría realizada por la Dirección Académica de sede, se lograron identificar beneficios para el mejoramiento de la calidad académica de los programas que hacen relevante el proceso de autoevaluación, al igual que se distinguieron dificultades que han retrasado el logro de los resultados esperados en el tiempo previsto, lo cual ha llevado a reprocesos y ejecución de recursos no contemplados.

Como beneficios identificados a partir de las prácticas adelantadas y de las experiencias obtenidas durante el desarrollo de los diferentes procesos de autoevaluación, se pueden resaltar los siguientes:

- Apuesta por una cultura de evaluación continua: Para la Universidad Nacional de Colombia, la evaluación continua "es el seguimiento anual de la calidad del programa con base en indicadores" (Art. $5^{\circ}$. Acuerdo 151 de 2014 del CSU). La revisión periódica a partir de los indicadores en el ejercicio de evaluación y seguimiento permite que los programas curriculares se involucren más en sus procesos y lo entiendan como un ambiente de mejoramiento permanente.

La Dirección Académica de la sede Manizales ha liderado la formulación de proyectos de inversión en los últimos tres trienios que han buscado consolidar una cultura de la autoevaluación y la evaluación continua en la comunidad académica.

- Implementación de una plataforma de autoevaluación para los programas curriculares. Este sistema agrupa la información de los indicadores requeridos para adelantar el proceso de autoevaluación y formular el plan de mejoramiento. La plataforma permite que los programas puedan mantener actualizada su información a modo de consulta y para la toma de decisiones.

- Acreditación de los programas curriculares y su posicionamiento en el medio. El desarrollo de las diferentes etapas del proceso de autoevaluación ha permitido lograr y evidenciar un reconocimiento de la calidad académica de los programas curriculares de la institución.

Fruto de estas iniciativas de evaluación, la sede Manizales cuenta con once (11) programas curriculares de pregrado 
acreditados de alta calidad (su totalidad), cinco (5) programas de posgrado (4 maestrías y un doctorado) con este reconocimiento, y cuatro programas de posgrado más que vienen adelantando el proceso.

- Formulación de proyectos de inversión para la mejora continua. La consolidación de los procesos de autoevaluación y acreditación desde los programas curriculares ha permitido identificar necesidades contempladas en los respectivos planes de mejoramiento. Desde ese punto de partida, la institución ha logrado formular proyectos que han tenido como objetivo la transformación de dichas necesidades en oportunidades de mejora. Entre otros, se pueden resaltar:

- Plan 150 X150: Fomento de la Cultura de Evaluación Continua a Través del Apoyo a Planes de Mejoramiento de los Programas Curriculares de la Sede Manizales en los 150 Años de Excelencia Académica en la Universidad Nacional De Colombia

- Internacionalización de los Programas Curriculares y el Fortalecimiento de la Movilidad Académica en los 150 Años de la Universidad Nacional de Colombia

- Fortalecimiento de la Calidad Académica de los Programas Curriculares a Través del Mejoramiento de los Espacios Académicos Transversales de Docencia de la Sede Manizales

- Acompañamiento Académico para Disminuir la Deserción de los Estudiantes de la Universidad Nacional de Colombia

Así, teniendo como referente la concepción de estrategia en el marco de una organización, particularmente en el sector educativo, estas pueden volverse cruciales si se implementan correctamente dado que tienen relación con el quehacer de la organización con base en su estructura, que tiene relación con la acumulación de experiencias y que está determinado a un patrón de acciones que se implementan en el tiempo.

Resultado de la revisión realizada de los últimos informes de autoevaluación elaborados por los programas de pregrado de la Facultad de Administración de la Universidad Nacional de Colombia, referentes de la investigación, se logró identificar e interpretar la relación con la gestión directiva y académica según el MEN y diferentes autores.

Teniendo este insumo y la identificación y caracterización de los mismos procesos, se lograron definir algunas propuestas estratégicas que están dirigidas a la consolidación de una cultura de la autoevaluación.

\section{Recomendaciones desde la Gestión Directiva}

Desde la gestión directiva, vista como el direccionamiento y la gestión estratégica en una institución educativa, se dispusieron las siguientes propuestas:

1. Implementación de políticas públicas transversales que den cuenta del fortalecimiento de los ejes misionales de la institución: Tener claridad sobre la realidad institucional, desde su gestión académica y directiva, puede propiciar oportunidades para la formulación de proyectos transversales con visión a largo plazo.

2. Relacionamiento con el sector externo (empresa-estado-comunidad) para la generación de alianzas: constituye la oportunidad de articular diferentes actores (directivos, docentes, estudiantes y administrativos) tanto internos como externos para el cumplimiento de los objetivos misionales, al poder considerar aspectos como la pertinencia y relevancia social.

3. Vinculación y capacitación de la comunidad académica (estudiantes, profesores, egresados y administrativos): El ejercicio de evaluación debe propiciar espacios con los diferentes protagonistas en que 
confluyan puntos de vista, brindando una identidad a la gestión estratégica de los mismos programas.

4. Articulación de los sistemas de información para la toma de decisiones en pro de la consolidación y mejoramiento de los procesos de autoevaluación tanto institucional como de programas.

\section{Recomendaciones desde la Gestión Académica}

Desde la revisión y la relación provista en los informes de autoevaluación, se puede plantear la siguiente propuesta para los programas evaluados:

Caracterización y realimentación de las metodologías de enseñanza - aprendizaje: desde esta línea se puede considerar el diseño, creación y oferta de cursos de desarrollo profesoral, la creación de semilleros de innovación pedagógica y de aseguramiento de la calidad académica, la sistematización de experiencias y reflexiones profesorales y fortalecimiento de los procesos de acompañamiento estudiantil.

\section{Conclusiones}

Fruto de la investigación realizada desde la revisión de los informes de autoevaluación de los programas de pregrado de la Facultad de Administración, se pudo evidenciar y concluir que existe una convergencia entre algunos de los factores y características establecidos en los procesos de autoevaluación y los procesos y componentes definidos por el MEN para la gestión directiva y académica de una institución, los cuales asimilan su forma de acción e implementación de estrategias desde sus ejes misionales para el mejoramiento de los programas curriculares como de la misma institución, lo que el MEN considera como la Ruta del Mejoramiento. La Universidad Nacional de Colombia ha sido pionera al implementar un modelo de autoevaluación que define etapas, recursos y normatividad a partir de los parámetros establecidos por el CNA para tal fin, al considerar los procesos de autoevaluación como esfuerzos estratégicos que la siguen posicionando entre las mejores instituciones de educación superior del país.
Así, la gestión académica y directiva reflejada en los programas curriculares, desde la autoevaluación, ha facilitado la valoración y el mejoramiento continuo de sus procesos al obtener el reconocimiento de calidad de sus programas curriculares. Además, a través del ejercicio de gestión, la institución ha logrado implementar proyectos de inversión que han fortalecido el compromiso por generar una cultura de la evaluación y el mejoramiento continuo a partir de los procesos de autoevaluación de los diferentes programas.

El hecho de visibilizar la relación de la gestión académica y directiva con los procesos de autoevaluación, además de generar una cultura en la comunidad académica, permite la articulación entre los diferentes planes de acción de la institución, en sus diferentes niveles (Plan Global de Desarrollo, Planes de acción de sedes, de Facultad y planes de mejoramiento de programas). La sinergia entre gestión académica y directiva con los procesos de autoevaluación permite a su vez identificar líneas estratégicas proyectadas a mediano y largo plazo, que conllevan a la implementación y ejecución de acciones de alto impacto para la comunidad universitaria y para el medio externo.

\section{Referencias}

Bolseguí, M., y Fuguet Smith, A. (2006). Cultura de evaluación: Una aproximación conceptual. Investigación y Postgrado, 21(1), 77-98.

Casallas, P., y Gaona, J. (2013). La cultura organizacional, factor crítico de éxito en procesos de gestión de la calidad y de acreditación institucional. SIGNOSInvestigación en sistemas de gestión, 5(1), 15-30.

Camisón, C., Cruz, S., y González, T. (2006). Gestión de la calidad, conceptos, enfoques, modelos y sistemas. Madrid: Pearson Prentice Hall.

Congreso de Colombia. (28 de diciembre de 1992). Servicio público de la Educación Superior. [Ley 30 de 1992] Recuperado de https://www.mineducacion.gov.co/1621/ articles-86437_Archivo_pdf.pdf 
Consejo Nacional de Acreditación (CNA, 2013). Lineamientos Para La Acreditación De Programas De Pregrado.

Ehlers, U. D. (2010). Moving from control to culture in Higher Education Quality. Changing Cultures in Higher Education (pp. 385-401). Springer, Berlin, Heidelberg.

Ferrer, A. (2008). Evaluación y cambio de los sistemas educativos: la interacción que falta. Ensaio: avaliação e políticas públicas em educação,16(59), 275-296. https://dx.doi. org/10.1590/S0104-40362008000200007

Gimeno Sacristán, J. (1998). El curriculum. Una reflexión sobre la práctica. Madrid: Editorial Morata. Págs. 373-403.

González Consuegra, R. V. (1997). Educación superior: autonomía vs proceso de acreditación. Avances en Enfermería, 15(1-2), 23-27.

González, L. E. (2005). El impacto del proceso de evaluación y acreditación en las universidades de América Latina. Recuperado de https:// www.uned.ac.cr/academica/images/igesca/ materiales/12.pdf

Guerrero, O. (1980). La Administración Pública del Estado Capitalista. México: Ediciones INAP

Johnson, G., y Scholes, K. (1997). Dirección estratégica. Análisis de las estrategias de las organizaciones. $3^{a}$ ed. Madrid: Prentice Hall.

Lopera, C. (2004). Antinomias, dilemas y falsas premisas que condicionan la gestión universitaria. Revista Mexicana de Investigación Educativa, vol. 9, núm. 22, pp. 617-635. Consejo Mexicano de Investigación Educativa, A.C. Distrito Federal, México

Londoño, J., y Ramírez, D. (2012). Cultura de la autoevaluación: cimiento para la acreditación de programas académicos en modalidad virtual. Revista Virtual Universidad Católica del Norte, (36), 1-6.

Masland, A. T. (1985). Organizational culture in the study of higher education. The Review of Higher Education, 8(2), 157-168.

Ministerio de Educación Nacional [MEN] (2013). Estado del arte del sistema nacional de acreditación e identificación de rutas y tópicos de investigación y profundización para el mejoramiento de las condiciones de calidad. Bogotá: SECAB - Publicaciones.

Ministerio de Educación Nacional [MEN] (2010). Lineamientos generales para la autoevaluación en el marco del artículo 6, numeral 6.3 del decreto 1295 de 2010. Recuperado de https://www.mineducacion.gov.co/ sistemasdeinformacion/1735/article-298334. html

Ministerio de Educación Nacional [MEN] (2008). Guía para el mejoramiento institucional - de la autoevaluación al plan de mejoramiento. Guía No. 34.

Mintzberg, H., Quinn, J. B., y Voyer, J. (1997). El proceso estratégico: conceptos, contextos y casos. México: Pearson Educación

Montoya Restrepo, I. A. (2009). La formación de la estrategia en Mintzberg y las posibilidades de su aportación para el futuro. Revista Facultad de Ciencias Económicas: Investigación y Reflexión, 17(2), 23-44.

Orozco, L., y Cardoso, R. (2003). La evaluación como estrategia de autorregulación y cambio institucional. Perfiles educativos, 25(102), 7382.

Pabón, N. (2001). La acreditación en Colombia: balance de cinco años. Revista de la Educación Superior en Línea. (119)

Presidencia de la República de Colombia (25 de julio de 2019) Artículo 1. Por el cual se sustituye el Capítulo 2 y se suprime el Capítulo 7 del Título 3 de la Parte 5 del Libro 2 del Decreto 1075 de 2015 -Único Reglamentario del Sector Educación. [Decreto 1330 de 2019] Recuperado de https://www.mineducacion. gov.co/1759/articles-387348_archivo_pdf.pdf 
Presidencia de la República de Colombia (31 de diciembre de 1994) Por el cual se reglamentan los artículos 53 y 54 de la Ley 30 de 1992. [Decreto 2904 de 1994] Recuperado de http:// wWW. suin-juriscol.gov.co/viewDocument. asp?ruta=Decretos $/ 1500345$

Rangel, H. (2010). Hacia una evaluación generadora: Más allá de la evaluación técnicoburocrática de las universidades en México. Revista Iberoamericana de Educación, 54(1), 6.

Reyes, C. I. (2006). La nueva cultura de la evaluación en educación superior. En IV Congreso Internacional de Docencia Universitaria e Innovación, Barcelona (Vol. 30).

Silva, J., Bernal, E., y Hernández, C. (2014). Modelo de aseguramiento interno de la calidad para las instituciones de educación superior en el marco del mejoramiento continuo de la calidad de la educación superior en Colombia. Ministerio de Educación Nacional. Recuperado de https://www. cna.gov.co/1741/articles-186502_Modelo_ aseguramiento.pdf

Universidad Nacional de Colombia sede Manizales (2015). Informe de autoevaluación para renovación de acreditación del programa de Administración de Sistemas Informáticos. Facultad de Administración.

Universidad Nacional de Colombia. (2014). Normalización del proceso de autoevaluación y seguimiento de la calidad de los programas curriculares de la Universidad Nacional de Colombia. [Acuerdo 151 de 2014 del Consejo Superior Universitario]. Recuperado de http:// www.legal.unal.edu.co/rlunal/home/doc. jsp?d_i=66353

Universidad Nacional de Colombia sede Manizales (2014). Informe de autoevaluación para renovación de acreditación del programa de Administración de Empresas. Facultad de Administración.

Universidad Nacional de Colombia sede Manizales (2014). Informe de autoevaluación para renovación de acreditación del programa de Gestión Cultural y Comunicativa. Facultad de Administración.

Universidad Nacional de Colombia (2012). Guía para consolidar el Proyecto Educativo de Programa - PEP. Vicerrectoría Académica. Dirección Nacional de Programas de Pregrado.

Universidad Nacional de Colombia (2010). Guía de autoevaluación y seguimiento de la calidad programas de pregrado. Vicerrectoría Académica. Dirección Nacional de Programas de Pregrado. Primera Edición.

Valenzuela, J., Ramírez, M., y Alfaro, J. (2011). Cultura de evaluación en instituciones educativas: Comprensión de indicadores, competencias y valores subyacentes. Perfiles educativos, 33(131), 42-63. Recuperado de http://www.scielo.org.mx/ scielo. php?script=sci_arttext\&pid=S018526982011000100004\&lng=es\&tlng=es. 